\begin{tabular}{|c|l|}
\hline Title & Influence of specific heats on indicator diagram analysis in a hydrogen-fuelled SI engine \\
\hline Author(s) & Shudo, Toshio; Nabetani, Shigeki; Nakajima, Y asuo \\
\hline Citation & $\begin{array}{l}\text { JSAE Review, 22(2), 224.226 } \\
\text { https://doi.org/10.1016/S0389-4304(01)00094-7 }\end{array}$ \\
\hline Issue Date & 2001-04 \\
\hline Doc URL & http://hdl.handle.net/2115/32326 \\
\hline Type & article (author version) \\
\hline File Information & JSAE Review 22-2.pdf \\
\hline
\end{tabular}

Instructions for use 


\section{Influence of Specific Heats on Indicator Diagram Analysis in a Hydrogen-Fuelled SI Engine}

\section{Toshio SHUDO}

Applied Energy System Group, Division of Energy and Environmental Systems, Hokkaido University

N13 W8, Kita-Ward, Sapporo, Hokkaido, 060-8628, JAPAN

Email: shudo@eng.hokudai.ac.jp

Tel/Fax: +81-11-706-6402

\section{Shigeki NABETANI}

ZEXEL Corporation

\section{Yasuo NAKAJIMA}

Musashi Institute of Technology

\section{Introduction}

Hydrogen has been investigated as a clean alternative to fossil fuel. However, hydrogen has unique characteristics in combustion which influence the thermal efficiency of internal combustion engines. Namely, hydrogen has a higher flame propagation velocity and a shorter quenching distance as compared with hydrocarbons [1]. The combustion characteristics of hydrogen supposedly influence the cooling loss and the degree of constant volume. The cooling loss and the degree of constant volume are two major factors influencing the thermal efficiency of internal combustion engines. Authors have investigated the influences of the cooling loss ratio and the degree of constant volume on thermal efficiency of a hydrogen-fuelled SI engine in the previous research. In the research, the cooling loss ratio was quantitatively evaluated by using analyses of indicator diagram and exhaust gas composition [2], and the degrees of constant volume cooling and constant volume burning were also evaluated from indicator diagram [3]. This research analyzed influences of treatment of the specific heats on the indicator diagram analysis in a hydrogen-fuelled SI engine.

\section{Experimental apparatus}

The engine tested in this research was a 4-stroke 4-cylinder spark ignition engine (bore: $85 \mathrm{~mm}$, stroke: $88 \mathrm{~mm}$, compression ratio: 8.5). Fuel gas was measured with a mass flow meter (Oval F203S) and continuously supplied into intake manifold. In-cylinder pressure was measured with a piezoelectric type pressure transducer (AVL, GM12D) installed on the cylinder head. 200 cycles of pressure data were averaged and used to calculate the indicated thermal efficiency, the apparent rate of heat release, the degree of constant volume, and others. The oxygen concentration in the exhaust gas was measured with an MPD type analyzer and used to calculate the combustion efficiency.

\section{Results and discussions}

The apparent rate of heat release $d Q / d \theta$ in the internal combustion engines can be described with in-cylinder volume $V$, in-cylinder pressure $P$, ratio of specific heats $\kappa$, and crank angle $\theta$, as follows.

$$
d Q / d \theta=(V d P / d \theta+\kappa P d V / d \theta) /(\kappa-1)-P V(\kappa-1)^{2} d \kappa / d \theta
$$

The third term in the right side of the equation, $P V(\kappa-1)^{2} d \kappa / d \theta$, is quite small in conventional gasoline or diesel engines and sometimes neglected in combustion analyses. Although the ratio of specific heats in hydrogen combustion is not so different from that in hydrocarbon combustion, a change in the ratio of specific heats is supposedly faster than hydrocarbon combustion because of the high burning velocity of hydrogen. This research 
analyzed the influence of the treatment of the ratio of specific heats on combustion analysis in a hydrogen-fuelled SI engine. Figure 1 shows the comparison among results of the following three cases of calculations.

Case 1: The ratio of specific heats $\kappa$ is calculated from composition and temperature of the in-cylinder gas for each crank angle $\theta$ and treated as a variable in Eq.(1).

Case 2: The ratio of specific heats $\kappa$ is calculated with the same method as the case 1 and treated as a variable in the following equation, i.e. the term $P V(\kappa-1)^{2} d \kappa / d \theta$ is neglected.

$$
d Q / d \theta=(V d P / d \theta+\kappa P d V / d \theta) /(\kappa-1)
$$

Case 3: The ratio of specific heats $\kappa$ is treated as a constant value 1.4 in Eq.(2).

The figure shows the comparison of the results of the above three methods in the calculation of the apparent rate of heat release. Results of methane combustion are also shown in the figure for a reference. The negative heat release is observed after the end of combustion, because the apparent rate of heat release is affected by the cooling loss to cylinder walls. The results show that simpler calculation method in the three cases has both smaller negative heat release and smaller value of the maximum apparent rate of heat release $d Q / d \theta$.

Figure 2(a) shows the maximum values of the apparent rate of heat release $d Q / d \theta_{\max }$ and the $d \kappa / d \theta$ versus the ignition timing. In both fuels, the simpler calculation without the term $P V(\kappa-1)^{2} d \kappa / d \theta$ decreases the $d Q / d \theta_{\max }$. The difference between the results of the two cases of calculations is larger in hydrogen combustion especially at the advanced ignition timings. The faster change in the ratio of specific heats $\kappa$ due to the rapid change in composition and temperature of the in-cylinder gas due to the higher burning velocity for hydrogen supposedly influences results of the apparent rate of heat release. Figure 2(b) shows the degree of constant volume $\eta_{g l h}$ and the cooling loss ratio $\phi_{w}$ against the ignition timing. The degree of constant volume $\eta_{g l h}$ was calculated with the apparent rate of heat release $d Q / d \theta$ in the following equation.

$$
\eta_{g l h}=1 /\left(\eta_{t h} Q\right) \int\left(1-\left(\left(V_{h}+V_{c}\right) / V(\theta)\right)^{1-\kappa}\right) d Q / d \theta d \theta
$$

The cooling loss ratio $\phi_{w}$ was evaluated with the cumulative apparent heat release $Q$, the heat of fuel supplied in a cycle $Q_{\text {fuel }}$ and the combustion efficiency $\eta_{u}$ as follows [2]-[3].

$$
\begin{aligned}
\phi_{w} & =Q_{C} / Q_{B} \\
Q_{C} & =Q_{B}-Q \\
Q_{B} & =\eta_{u} Q_{\text {fuel }} \\
\phi_{w} & =1-Q /\left(\eta_{u} Q_{\text {fuel }}\right)
\end{aligned}
$$

Here, $Q_{B}$ and $Q_{C}$ stand for the cumulative real heat release and the cumulative cooling loss respectively.

The result shows that the neglect of the $d \kappa / d \theta$ hardly influences the degree of constant volume $\eta_{g l h}$. However, the cooling loss ratio $\phi_{w}$ derived from the apparent rate of heat release $d Q / d \theta$ is largely affected by the treatment of the ratio of specific heats $\kappa$ in the calculation.

Figure 3 shows results of similar analyses as functions of the excess air ratio. The cooling loss ratio $\phi_{w}$ derived from the apparent rate of heat release $d Q / d \theta$ is largely influenced by the treatment of the ratio of specific heats $\kappa$. The influence is larger especially in smaller excess air ratio with higher burning velocity.

From the above results, the neglect of the $d \kappa / d \theta$ in the calculation of the apparent rate of heat release $d Q / d \theta$ has almost no influence on the calculated degree of constant volume $\eta_{g l h}$. However, the neglect has large influence on the cooling loss ratio $\phi_{w}$ calculated from the apparent rate of heat release $d Q / d \theta$, especially in hydrogen combustion with higher burning velocity and faster change in specific heats during combustion period. Therefore, the $d \kappa / d \theta$ should not be neglected in the calculation of apparent rate of heat release $d Q / d \theta$ in combustion analysis of hydrogenfuelled engines.

\section{Conclusion}

This research analyzed influences of treatment of the ratio of specific heats $\kappa$ on the calculated apparent rate of heat release. The results derived from the analysis are:

(1) Hydrogen combustion has larger $d \kappa / d \theta$ during combustion period than hydrocarbon combustion because of the 
faster change in composition and temperature of the in-cylinder gas due to the higher burning velocity for hydrogen;

(2) The $d \kappa / d \theta$ in the hydrogen combustion is especially large in the operation at advanced ignition timing and at the excess air ratio close to stoichiometric; and

(3) The analysis of the cooling loss ratio using the apparent rate of heat release should not neglect the $d \kappa / d \theta$ especially in hydrogen-fuelled engines.

\section{References}

[1] Lewis, B., et al.: Combustion, Flames and Explosions of Gases, Academic Press, pp.331-335(1961).

[2] Shudo, T., et al.: Thermal Efficiency Analysis in a Hydrogen Premixed Combustion Engine, JSAE Review, Vol.21, No.2, pp.177-182(2000).

[3] Shudo, T., et al.: Analysis of Degree of Constant Volume and Cooling Loss in a Hydrogen Premixed Combustion Engine (in Japanese with English summary), Transaction of JSAE, Vol.31, No.4, pp.5-10(2000).

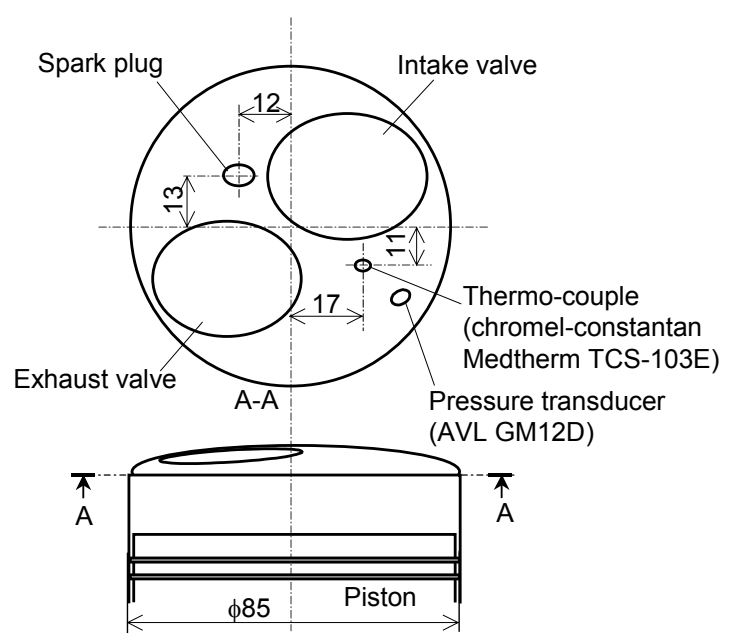

Fig.1 Combustion chamber geometry of tested engine 


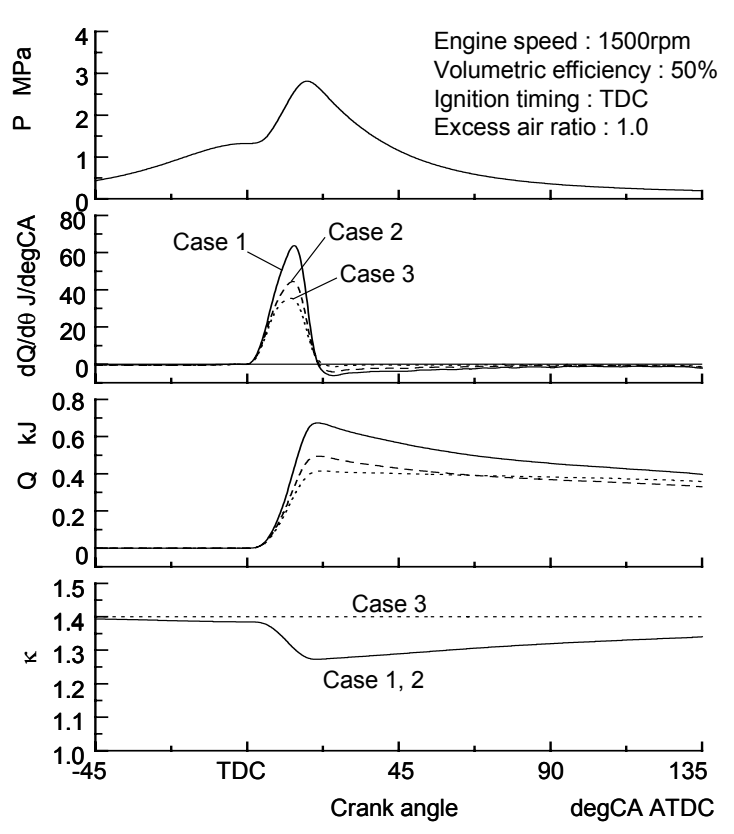

(a) Hydrogen combustion

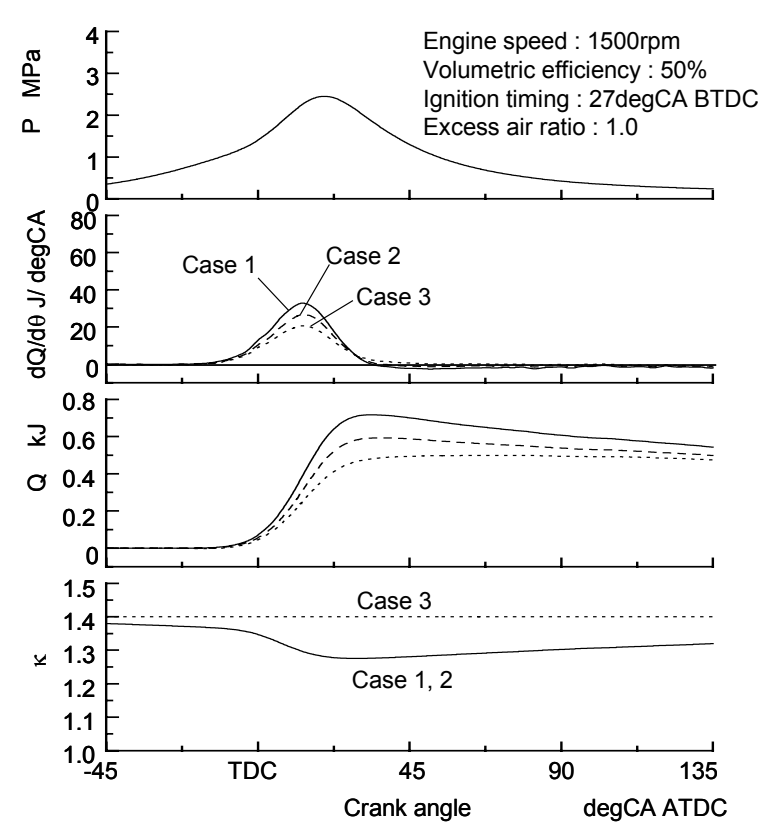

(b) Methane combustion

Fig.2 Influence of calculation method of heat release rate

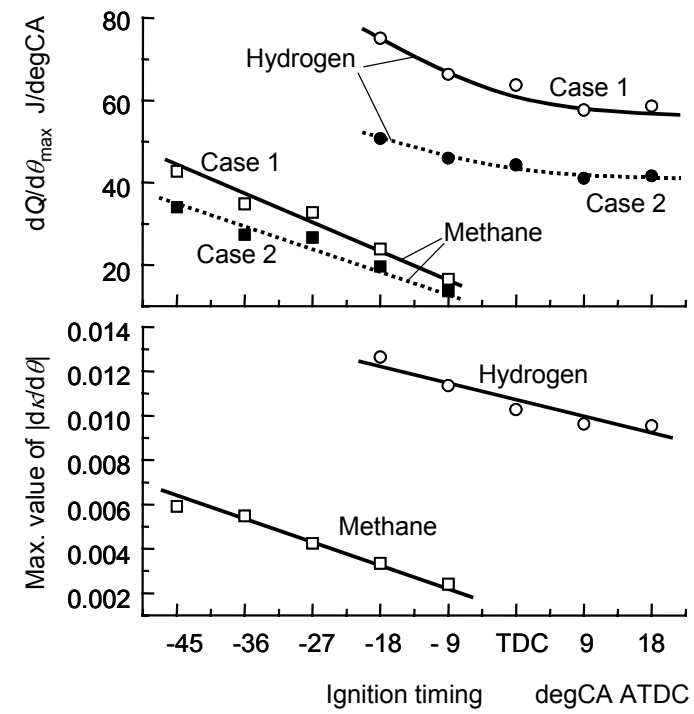

(a) Maximum value of $d Q / d \theta$ and $|d \kappa / d \theta|$

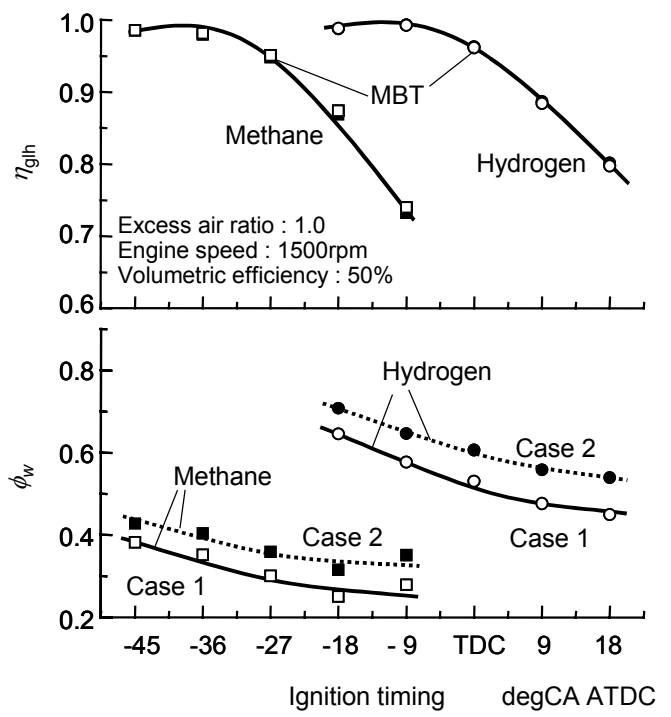

(b) Degree of constant volume and cooling loss ratio

Fig. 3 Influence of calculation method of heat release rate 


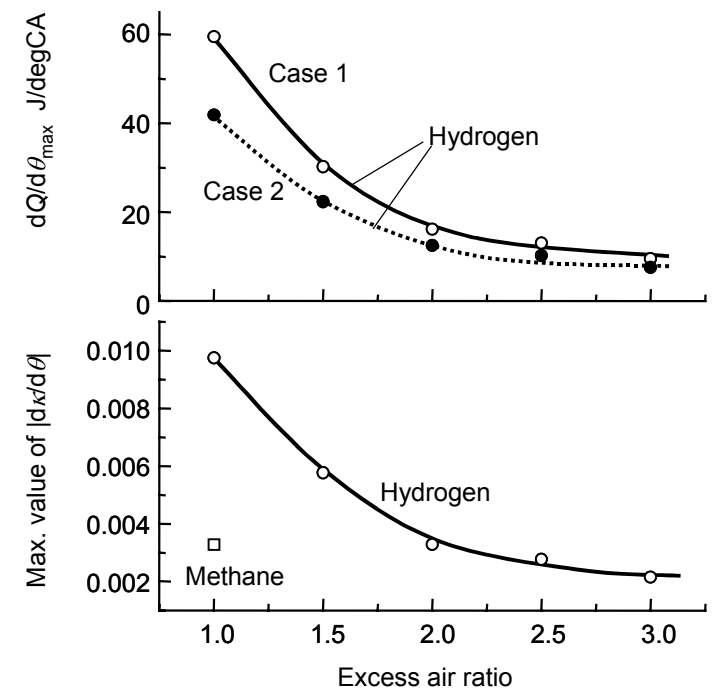

(a) Maximum value of $d Q / d \theta$ and $|d \kappa / d \theta|$

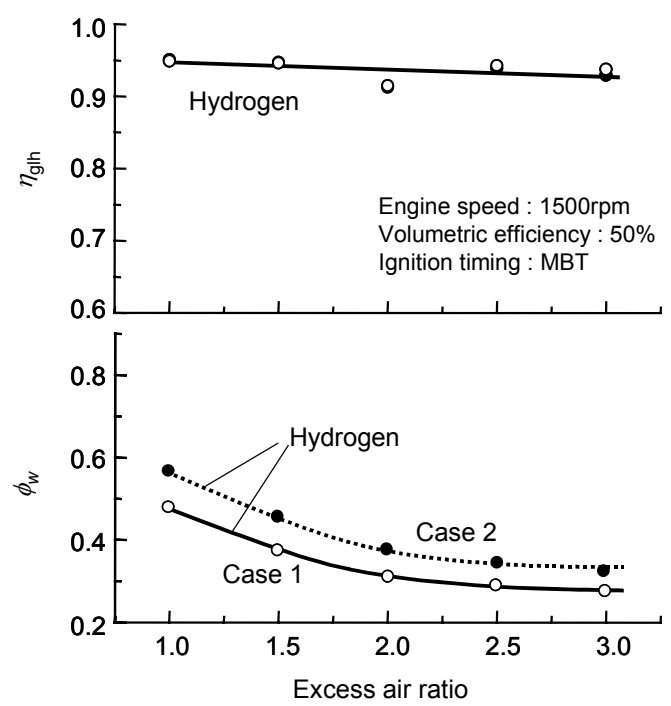

(b) Degree of constant volume and cooling loss ratio

Fig. 4 Influence of calculation method of heat release rate
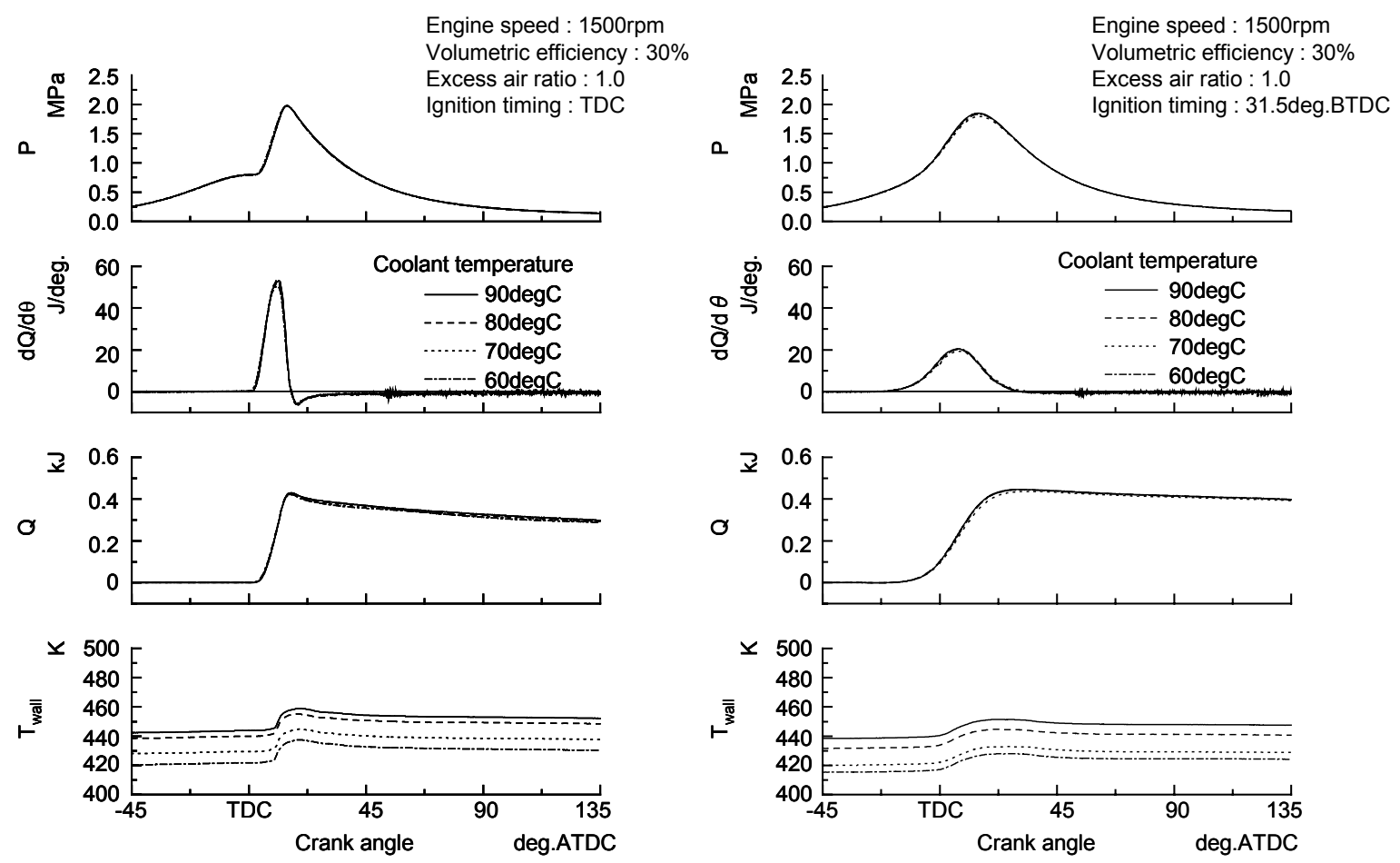

(a) Hydrogen combustion

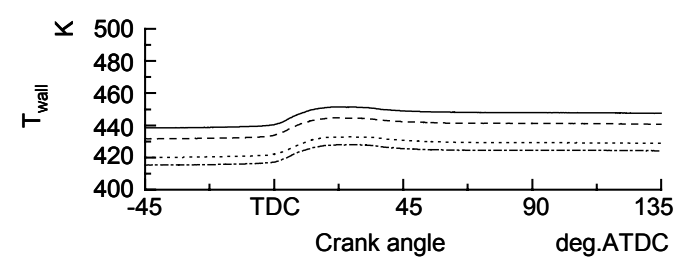

(b) Methane combustion

Fig. 5 Influence of coolant combustion and combustion chamber wall temperature 


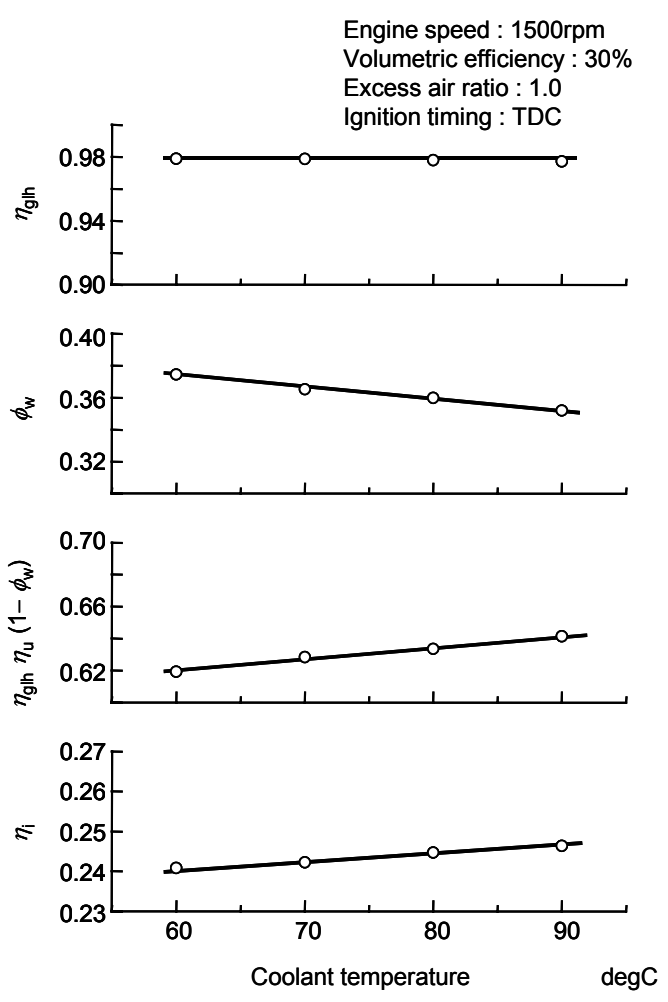

(a) Hydrogen combustion

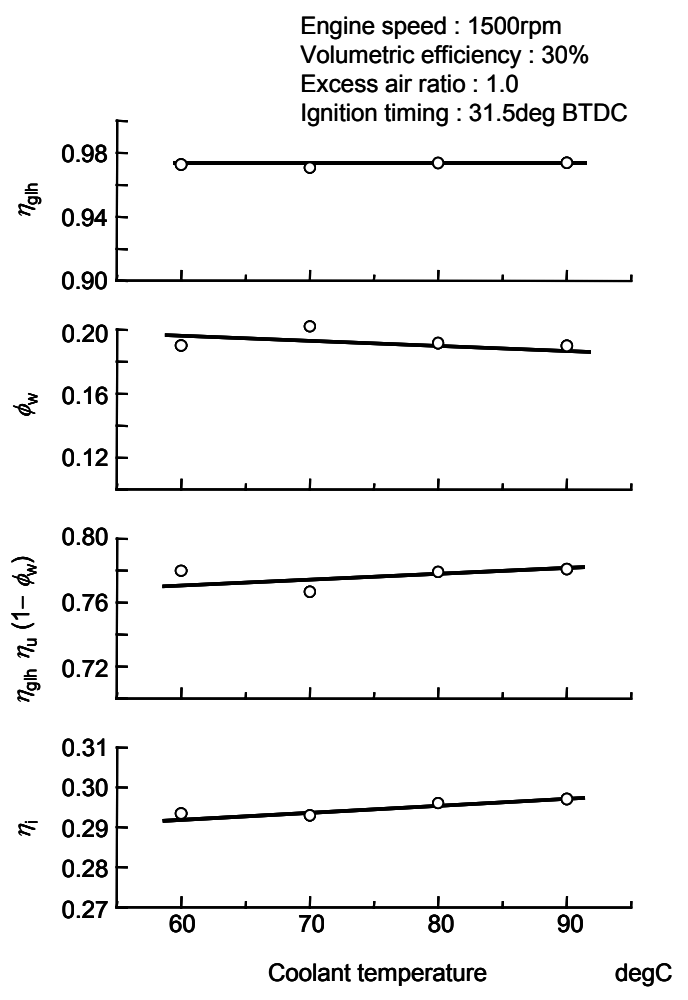

(b) Methane combustion

Fig.6 Influence of coolant temperature on thermal efficiency

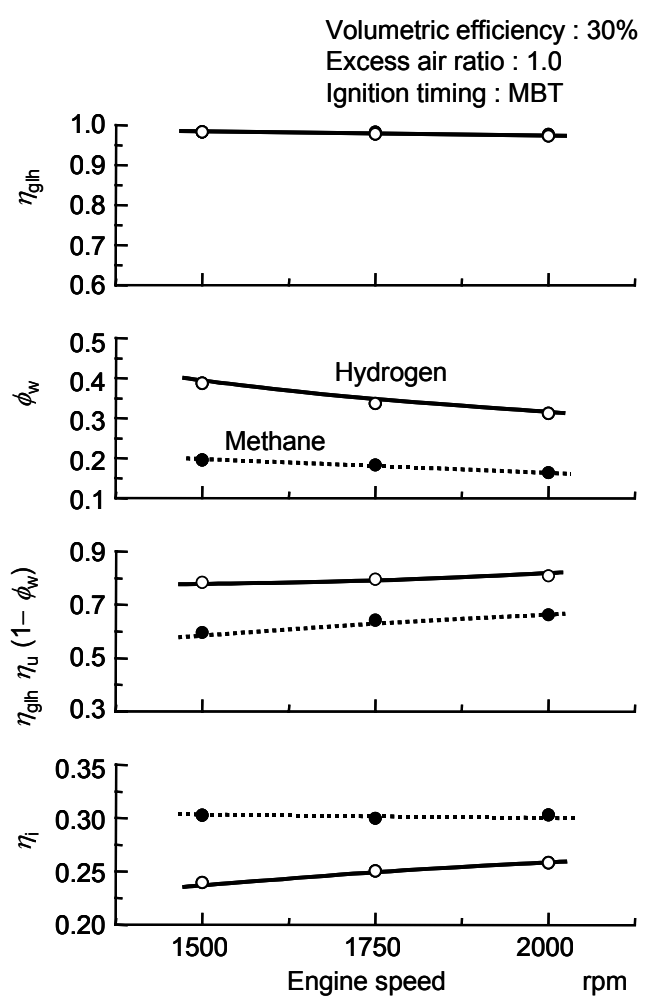

Fig. 7 Influence of engine speed on thermal efficiency 